Discrete Comput Geom 34:537-544 (2005)

DOI: $10.1007 / \mathrm{s} 00454-005-1178-\mathrm{y}$

\title{
A New Packing Density Bound in 3-Space
}

\author{
Edwin H. Smith \\ MCIS Department, Jacksonville State University, \\ Jacksonville, AL 36265, USA \\ esmith@jsucc.jsu.edu
}

\begin{abstract}
It is shown that every compact convex set $K$ which is centrally symmetric and has a non-empty interior admits a lattice packing of Euclidean 3 -space with density greater than or equal to $0.53835 \ldots$. This is an improvement of the result in [8], which achieved a bound of $0.46421 \ldots$. Minkowski combinations and the Brunn-Minkowski inequality are used in conjunction with the construction in [8] to achieve a better result.
\end{abstract}

\section{Introduction}

A convex body is a compact convex set having a non-empty interior. In the context of this paper we are only concerned with convex bodies in Euclidean 3-space $\left(E^{3}\right)$ and in the Euclidean plane $\left(E^{2}\right)$ and denote by $K$ an arbitrary convex body. A convex body in the plane is referred to as a convex disk. $K$ is centrally symmetric if there exists a point $o$ in $K$ such that $o$ is the midpoint of every chord of $K$ which contains $o$.

A packing with copies of $K$ is a family $\left\{K_{i}\right\}$ of sets congruent to $K$ whose interiors are mutually disjoint. A covering with copies of $K$ is a family $\left\{K_{i}\right\}$ of sets congruent to $K$ whose union is the entire space. A family $\left\{K_{i}\right\}$ which is both a packing and a covering is called a tiling. Any convex body which admits a tiling is called a convex tile.

Suppose $T$ is a tile and $T$ contains (respectively is contained in) $K$. If $\left\{T_{i}\right\}$ is a tiling with copies of $T$, then each $T_{i}$ contains (is contained in) a copy $K_{i}$ of $K$. Then $\left\{K_{i}\right\}$ is a packing (covering) associated with the tiling $\left\{T_{i}\right\}$.

Throughout this paper the measure (volume in 3-space or area in the plane) of any set $S$ will be denoted by $|S|$. Also, the length of any segment $s$ will be denoted $|s|$. Each packing (covering) is assigned a non-negative number, called the density of the packing (covering), which, intuitively, corresponds to the ratio of the sum of the measures of the bodies in the packing (covering) to the measure of the region which they pack (cover). For a formal definition of density and an investigation concerning its existence and uniqueness the reader is referred to Section III in Chapter 1 of [4]. The density of a 
tightest packing of Euclidean space with copies of $K$ is called the packing density of $K$. Likewise, the density of a thinnest covering of Euclidean space with copies of $K$ is called the covering density of $K$. The density of a most efficient packing (covering) with copies of $K$ by a lattice arrangement is the lattice packing (covering) density of $K$. In the case that the packing (covering) $\left\{K_{i}\right\}$ is associated with the tiling $\left\{T_{i}\right\}$, the density of $\left\{K_{i}\right\}$ can be computed as $|K| /|T|$. In general, if $\left\{K_{i}\right\}$ is a packing (covering) and $\left\{T_{i}\right\}$ is a tiling $(i=1,2,3, \ldots)$ and there is a number $r$ such that for every positive integer $i$ the distance between $K_{i}$ and $T_{i}$ is less than $r$, then the density can be computed as $|K| /|T|$. Therefore, if $\left\{K_{i}\right\}$ is a packing (covering) formed by translating copies of $K$ into a lattice arrangement, then the density can be computed as $|K| /|T|$ where $T$ is the lattice parallelepiped.

In this paper we investigate the following problem: find the greatest number $d_{0}$ such that every centrally symmetric convex body admits a lattice packing of Euclidean 3 -space with density greater than or equal to $d_{0}$. We will show that $d_{0} \geq 0.53835 \ldots$ A more general and difficult problem is to find the greatest such number $d_{0}$ for an arbitrary convex body. To achieve this bound, the construction in [8] is used, in which the author shows that every centrally symmetric convex body in $E^{3}$ will pack space with a density of at least $0.46421 \ldots$. This construction was also used in [9] in which the author bounds the ratio between packing and covering densities for a centrally symmetric convex body in $E^{3}$.

The theorem of Minkowski-Hlawka [5] gives a bound for the lattice packing densities of centrally symmetric convex bodies in dimension $d$, specifically $\zeta(d) / 2^{d-1}$ where $\zeta(d)=\sum_{k=1}^{\infty} k^{-d}$. For example, this theorem gives a density bound in $E^{3}$ of $0.30051 \ldots$ The density bound given in this paper, while much higher, is only for dimension 3 . It is likely that the greatest lower bound in this context is still significantly higher, since, for example, there is a lattice packing with congruent spheres which has density $\pi / \sqrt{18}=0.74048 \ldots$

The packing problem for centrally symmetric convex disks in $E^{2}$ has received much attention due to its role in the Geometry of Numbers and the bounds achieved leave much less room for improvement than the one shown here. Tammela [10] has shown that any centrally symmetric convex disk admits a packing of the plane with density greater than or equal to $0.89265 \ldots$. This bound was obtained through an elaboration of a method of Ennola [2] who suggested this improvement of his own result. However, it is conjectured that the "smoothed octagon" of Reinhardt [7], for which the packing density equals $0.90241 \ldots$, provides the best bound in this context. Furthermore, it is known that the maximum density for a centrally symmetric convex disk $K$ can be achieved by a lattice arrangement. This is a consequence of two theorems: The first by Dowker [1] asserts that among the minimum area hexagons containing $K$ there is one which is centrally symmetric about the center of $K$. The second by L. Fejes Toth [3] states that if $K$ is an arbitrary convex disk and $H$ is a hexagon of minimum area circumscribed about $K$, then the greatest packing density possible for $K$ is less than or equal to $|K| /|H|$. Since every centrally symmetric hexagon tiles the plane in a lattice manner, the greatest packing density for a centrally symmetric disk $K$ can be achieved by a lattice arrangement.

In this note the density bound given in [8] is improved by use of Minkowski combinations and the Brunn-Minkowski inequality. Though both of these apply to $E^{d}$, we only state and apply them in the context of $E^{2}$. 
Given two compact convex sets $K$ and $L$ in $E^{2}$, and $a, b \geq 0$, denote

$$
a K+b L=\{a x+b y \mid x \in K, y \in L\} .
$$

The convex set $a K+b L$ is called a Minkowski combination of the sets $K$ and $L$. The Brunn-Minkowski inequality asserts that the square root of area is a concave function with respect to Minkowski sums, that is, for $0 \leq x \leq 1$,

$$
\sqrt{|(1-x) K+x L|} \geq(1-x) \sqrt{|K|}+x \sqrt{|L|} .
$$

When the sets have non-empty interiors (i.e., they are convex disks), equality will hold if and only if $K$ and $L$ are homothetic, that is they differ by translations and/or dilations. In a classic result (for volumes in dimension $d$ ) due to Minkowski [6], it is shown that areas of Minkowski linear combinations can be represented by polynomials of the form

$$
|a K+b L|=a^{2}|K|+2 a b|(K, L)|+b^{2}|L|,
$$

where the coefficient $|(K, L)|$ is called the mixed area of $K$ and $L$. The above equation with the Brunn-Minkowki inequality implies Minkowski's inequality which assures

$$
|(K, L)| \geq \sqrt{|K|} \sqrt{|L|}
$$

with equality only when $K$ and $L$ are homothetic.

\section{The Theorem}

Theorem. If $K$ is a centrally symmetric convex body in $E^{3}$, then $K$ admits a packing of $E^{3}$ with density greater than or equal to $0.53835 \ldots$

Proof. We begin by constructing a packing of $E^{3}$ with copies of $K$. For simplicity, we assume that $K$ is strictly convex and has a smooth boundary, since any convex body can be approximated arbitrarily closely by a smooth, strictly convex body. Let $d$ be a chord in $K$ of maximum length. This chord must contain the center of symmetry. Otherwise, there would be two such parallel chords which would imply the existence of a longer chord. Let $L$ and $N$ be planes of support of $K$ such that they each contain an endpoint of $d$. Observe that $d$ is normal to both planes, since otherwise it would not be of maximum length. As a matter of convenience, we say that the orientation of the planes is horizontal and the orientation of $d$ is vertical. Let $o$ be the center of symmetry for $K$ and let $M$ be the plane parallel to $L$ which contains $o$. Let $C$ be the intersection of $M$ with $K$.

Now we pack the plane $M$ with copies of $C$ in a lattice arrangement so that the density of this packing is maximal among all packings. Before we proceed, we prove the following claim:

Claim. If $\left\{C_{i}\right\}$ is a maximal density lattice packing of $M$ with copies of $C$, then each copy of $C$ touches exactly six other copies. 


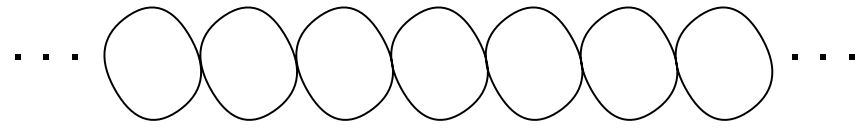

Fig. 1. A row of translates of $C$.

Proof. We assume we have a lattice packing of maximal density. Since the density is as large as possible, it is obvious that $C$ must touch a copy of itself. The vector $\vec{v}$ connecting their centers is a lattice vector, therefore there is a linear row of translates of $C$ such that each touches two others, as seen in Fig. 1. The packing of $M$ must consist of parallel rows identical to this one. Since $C$ is strictly convex, it is clear that $C$ cannot touch more than two copies of itself in the adjacent row. Assume that $C$ touches only one translate of itself in the adjacent row. Then this row can be shifted a small amount in the direction of $\vec{v}$ or $-\vec{v}$ so that $C$ does not contact it (see Fig. 2). Now the rows can be pushed closer together which increases the density of the packing. Therefore, if the packing has maximal density, $C$ must contact two members of each adjacent row as well as two members of its own row. Thus the claim is proven.

Shown in Fig. 3 are $C$ and its translates in the lattice packing of $M$ with their centers at the points of the lattice. We now consider a lattice arrangement of copies of $K$ such that each copy of $C$ is a cross section of a copy of $K$. Assume that $C$ and one of its translates $C^{\prime}$ in this arrangement have a boundary point $p$ in common. $K^{\prime}$ is the translate of $K$ in this arrangement which contains $C^{\prime}$. Because of symmetry, $p$ must be the midpoint of the segment which joins the center of $K$ with the center of $K^{\prime}$. Furthermore, the support plane of $K^{\prime}$ containing $p$ must be parallel to the support plane of $K$ at $p$. Thus the support planes coincide, which implies that the interior of $K$ has no point in common with the interior of $K^{\prime}$. Therefore, this arrangement of translates of $K$ between the planes $L$ and $N$ is a packing.

Our packing of $E^{3}$ will consist of copies of the layer which was just constructed. The layers in the packing will be translates of the first layer. This will be a lattice packing of $E^{3}$, therefore it will only be necessary to look at two adjacent layers in order to understand the entire packing. We denote the original lower layer $\Omega$ and the upper layer $\Omega^{*}$. We arrange the layers so that their supporting planes are parallel and so that $K$ in $\Omega$ touches its counterpart $K^{*}$ in $\Omega^{*}$. Seen in Fig. 4 are dots which represent the projections of segment $d$ and its copies onto the plane $M$. These points correspond to the extreme points or "peaks" in $\Omega$ on either side. Indicated by small circles are the projections of the peaks in $\Omega^{*}$ when they are pushed as far as possible into $\Omega$ without one copy of $K$

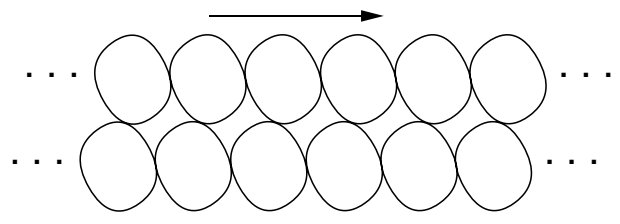

Fig. 2. Two rows which could be packed more closely. 


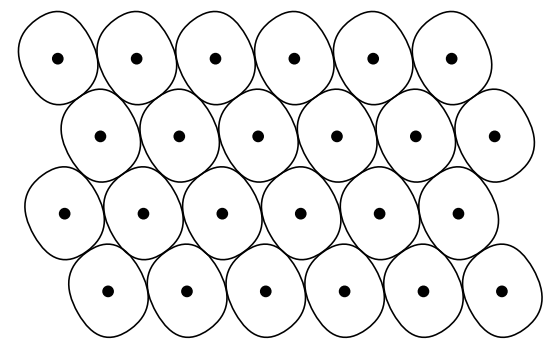

Fig. 3. A packing of $M$ with translates of $C$.

penetrating the interior of another. It is easy to observe that $K$ will now be touching at least three copies of itself in $\Omega^{*}$. Otherwise, $\Omega$ could be shifted within its supporting planes in some direction until $K$ does not touch any member of $\Omega^{*}$, allowing the layers to be pushed closer together. Since these are translates of a centrally symmetric convex body, each of these points of contact must be the midpoint of a segment which joins the center $o$ of $K$ with the center of some translate of $K$ in the other layer. Therefore their convex hull must lie in a plane which is parallel to $M$. The triangles formed by the points of contact between each member of $\Omega$ with members of $\Omega^{*}$ are seen in Fig. 5.

The triangle corresponding to $K$ is denoted $T_{K}^{1}$. Denote its reflection through the center of symmetry $o$ by $-T_{K}^{1}$. Due to the symmetry of $K$ and of the lattice parallelepiped, $\Omega^{*}$ will be a reflection of $\Omega$ through the midpoint of the segment joining the centers of $K$ and $K^{*}$. Therefore, $K^{*}$ will contain the reflection of triangle $T_{K}^{1}$ through one of its vertices. Figure 6 represents all such triangles in $\Omega^{*}$ (shaded), along with the triangles which were seen in Fig. 5. Notice that the sides of each triangle are parallel to the lattice vectors of a single layer and that each triangle has one-eighth of the area of the lattice parallelogram $P$.

The small circles in Fig. 7 indicate alternate locations for the peaks of $\Omega^{*}$ to be pushed into $\Omega$ so that each copy of $K$ touches three copies of $K$ in $\Omega$ (compare with Fig. 4). These points of contact between layers yield an analogous arrangement of congruent triangles identical to the arrangement in Fig. 6, but rotated $180^{\circ}$ about some point in one of the white triangles and possibly shifted a little (as seen in Fig. 8, with the lighter shading, superimposed upon the first arrangement). We denote the alternate triangle in $K$ by $T_{K}^{2}$. Of these two arrangements of the layers, we choose the one which allows the layers to penetrate each other more deeply, which we have previously assumed to be the

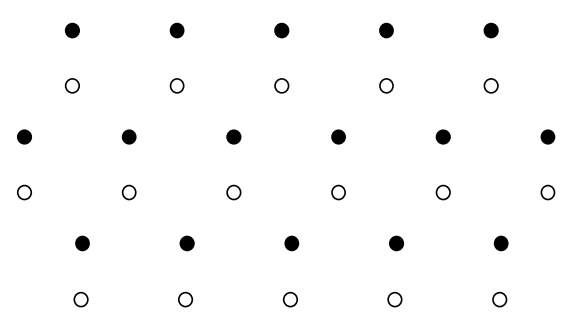

Fig. 4. Projections of the peaks of $\Omega$ and $\Omega^{*}$. 


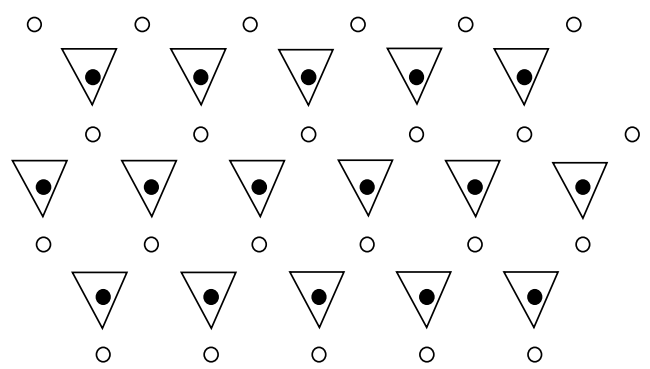

Fig. 5. Convex hulls of contact points for each translate in $\Omega$.

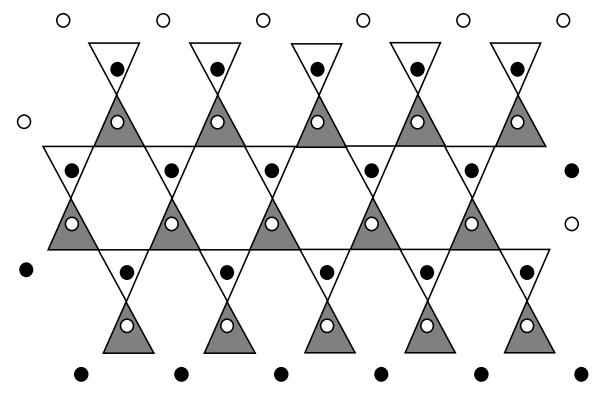

Fig. 6. The triangles of Fig. 5 with each of their reflections in $\Omega^{*}$.

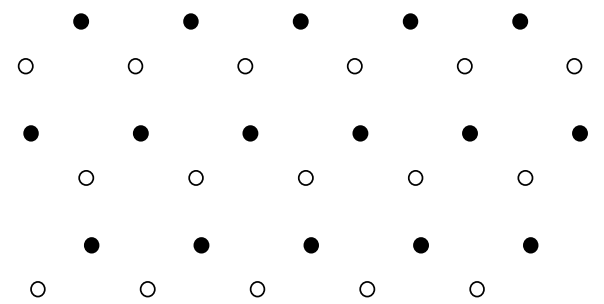

Fig. 7. Alternate locations for the peaks of $\Omega^{*}$.

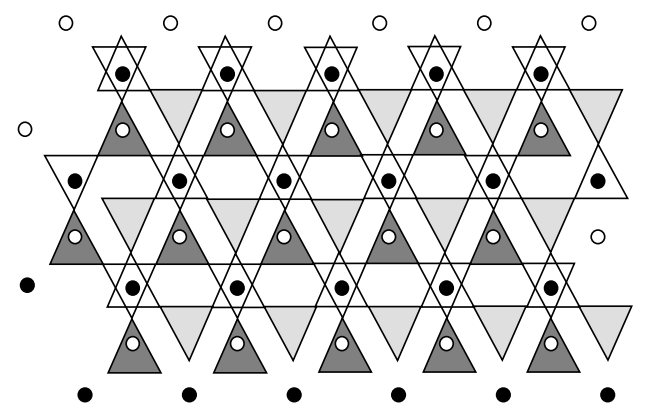

Fig. 8. The triangles for both the original and the alternate arrangements. 


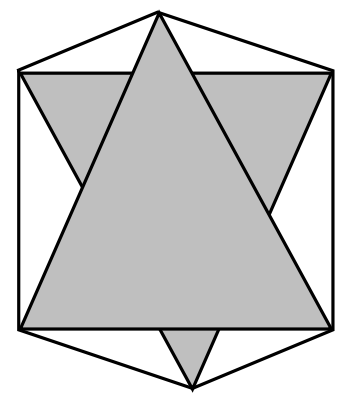

Fig. 9. The area of $H_{K}$ is computed.

first one. Then the convex hull of $T_{K}^{2}$ with $-T_{K}^{1}$ is a cylinder of triangular base. Its cross section in the plane containing $T_{K}^{1}$ is a translate of $T_{K}^{2}$. The convex hull of this cross section with $T_{K}^{1}$ is a hexagon $H_{K}$, possibly degenerated to a parallelogram. To compute its area, consider the arrangement of the triangles seen in Fig. 9. It is clear that the area of the convex hull is equal to the base of one triangle times its height. If a triangle is translated so that one of its vertices is in the interior of the other triangle, the area of the convex hull will increase. Consequently, the area of the hexagon is at least twice that of one triangle or a quarter of the area of the lattice parallelogram $P$.

Note that $H_{K}$ has its own center of symmetry. Then its reflection $-H_{K}$ through $o$ is identical and in a plane parallel to the plane which contains $H_{K}$. Let $h$ be the vertical distance between $H_{K}$ and $-H_{K}$. Notice that $h$ is also the vertical distance between the centers of the translates in adjacent layers. Therefore, the lattice parallelepiped in this packing has a volume of $h|P|$, implying a density of $|K| / h|P|$. K contains the convex hull of $C, H_{K},-H_{K}$, and $d$. We examine this convex hull more closely in order to establish a lower bound for $|K|$. To begin, we compute the volume of the convex hull of $C$ and $H_{K}$. The cross sections of this convex hull in planes parallel to $M$ will be all the Minkowski combinations of $C$ and $H_{K}$ of the form $x C+(1-x) H_{K}$ where $x$ ranges from 0 to 1 . Thus its volume is

$$
\frac{h}{2} \int_{0}^{1}\left(\left|x C+(1-x) H_{K}\right|\right) d x
$$

which by (1) is

$\frac{h}{2} \int_{0}^{1}\left(x^{2}|C|+x(1-x)\left|\left(C, H_{K}\right)\right|+(1-x)^{2}\left|H_{K}\right|\right) d x=\frac{h}{6}\left(|C|+\left|\left(C, H_{K}\right)\right|+\left|H_{K}\right|\right)$.

By Minkowki's inequality (2), this is greater than

$$
\frac{h}{6}\left(|C|+\sqrt{|C|} \sqrt{\left|H_{K}\right|}+\left|H_{K}\right|\right) .
$$

Therefore, the convex hull of $C, H_{K},-H_{K}$, and $d$ is greater than

$$
\frac{\left|H_{K}\right|(|d|-h)}{3}+\frac{h}{3}\left(|C|+\sqrt{|C|} \sqrt{\left|H_{K}\right|}+\left|H_{K}\right|\right) .
$$


Recalling that $\left|H_{K}\right|=|P| / 4$ and that this convex hull is in $K$, we have

$$
|K|>\frac{|P|(|d|-h)}{12}+\frac{h}{3}(|C|+\sqrt{|C|} \sqrt{|P| / 4 \mid}+|P| / 4) .
$$

Because of the affine invariant nature of this problem, we can assume $|P|=1$ and $|d|=1$. Then

$$
|K|>\frac{(1-h)}{12}+\frac{h}{3}\left(|C|+\frac{\sqrt{|C|}}{2}+\frac{1}{4}\right)
$$

That is,

$$
|K|>\frac{1}{12}+\frac{h|C|}{3}+\frac{h \sqrt{|C|}}{6} .
$$

Thus, we can say in regard to the density $D(=|K| / h|P|)$ of this packing,

$$
D>\frac{1}{12 h}+\frac{|C|}{3}+\frac{\sqrt{|C|}}{6} .
$$

Obviously, $D$ is minimized when $h$ is large. Since $h$ cannot be larger than $|d|$ (actually $h$ must be less than $|d|$ ), we minimize the density bound by setting $h=|d|=1$. Thus,

$$
D>\frac{1}{12}+\frac{|C|}{3}+\frac{\sqrt{|C|}}{6} .
$$

The copies of $C$ were packed in their plane with the greatest possible density. Therefore, by the result of Tammela [10] mentioned above, $|C| \geq 0.89265 \ldots$, which means that $D \geq 0.53835 \ldots$

This completes the proof of the theorem.

\section{References}

1. C. H. Dowker, On minimum circumscribed polygons, Bull. Amer. Math. Soc. 50 (1944), 120-122.

2. V. Ennola, On the lattice constant of a symmetric convex domain, J. London Math. Soc. 36 (1961), 135-138.

3. L. Fejes Toth, Some packing and covering theorems, Acta Sci. Math. Szeged 12/A (1950), 62-67.

4. L. Fejes Toth, Lagerungen in der Ebene, auf der Kugel und im Raum, Springer, Berlin, 1972.

5. E. Hlawka, Zur Geometrie der Zahlen, Math. Z. 49 (1944), 285-312.

6. H. Minkowski, Theorie der Konvexen Körper, insbesondere Begründung ihres Oberflachenbegriffs, in Gesammelte Abhandlungen von Hermann Minkowski (D. Hilbert, A. Speiser and H. Weyl, Eds.), Vol. 2, pp. 131-229, Teubner, Leipzig, 1911.

7. K. Reinhardt, Uber die dichteste gitterformige Lagerung kongruenter Bereiche in der Ebene und eine besondere Art konvexer Kurven, Abh. Math Sem. Hansischer Univ. 10 (1934) 216-230.

8. E. H. Smith, A density bound for efficient packings of 3-space with centrally symmetric convex bodies, Mathematika 46 (1999), 137-144.

9. E. H. Smith, A bound on the ratio between the packing and covering densities of a convex body, Discrete Comput. Geom. 23 (2000), 325-331.

10. P. Tammela, An estimate of the critical determinant of a two-dimensional convex symmetric domain (Russian), Izv. Vyssh. Ucebn. Zaved. Mat. 12 (1970), 103-107. 